\title{
Participants Perceptions of the effectiveness of CLEAR-AA's Development Evaluation Training Programme in Africa
}

\author{
${ }^{1}$ Mokgophana Ramasobana ${ }^{2}$ Mokgophana Ramasobana \\ Centre for Learning on Evaluation and Results- Wits University South Africa
}

\begin{abstract}
In the past few decades, M\&E capacity building programmes and training budgets aimed at addressing the rising demand for M\&E skills has been increasing. However, insufficient research assessing the effectiveness of these programmes have been conducted, therefore, their contribution towards building skills and knowledge is yet to be known. In this study, qualitative research aimed at assessing the effectiveness of capacity training interventions, particularly the Development Evaluation Training Programme in Africa (DETPA) delivered by CLEAR-AA was used as a case study. This is informed by DETPA's popularity in the region and afoot plans to implement the programme on an annual basis. Through semistructructured interviews, participant's perceptions on whether or not the DETPA programme has contributed towards building participant's individual capacities (skills and knowledge), their individual behavioural change, and organisational behavioural change as well as ascertained their perceptions regarding the gaps of programmes such as the 2017 DETPA were assessed. For the purpose of triangulation, interviews with ten (10) DETPA 2017 participants, one (1) DETPA facilitator, two (2) DETPA moderators and four (4) line managers of participants as the main data collection source were conducted. Generally, the findings indicate that there was positive feedback on the overall programme, improved skills and knowledge of participants, improved and sustained transfer of skills and knowledge and to some extent, the programme has contributed to change in behaviour in participant's respective organisations. Significantly, a contextually fitted curriculum content such as Made in Africa (MAE) was cited as one of the key findings that determined the perceived contribution of the DETPA programme.

This signifies the role of context in understanding the effectiveness of training programmes delivered in the African diaspora.
\end{abstract}

Keywords: evaluation capacity development, evaluation capacity building, human resource development, training and Made in Africa 